\title{
An Integrated Approach to Assertion-Based Random Testing in Prolog
}

\author{
Ignacio Casso $^{1(\bowtie)}$, José F. Morales ${ }^{1}$, Pedro López-García ${ }^{1,3}$, \\ and Manuel V. Hermenegildo ${ }^{1,2}$ \\ 1 IMDEA Software Institute, Madrid, Spain \\ \{ignacio.decasso, josef.morales, pedro. lopez, manuel.hermenegildo\}@imdea.org \\ 2 ETSI Informática, Universidad Politécnica de Madrid (UPM), Madrid, Spain \\ ${ }^{3}$ Spanish Council for Scientific Research (CSIC), Madrid, Spain
}

\begin{abstract}
We present an approach for assertion-based random testing of Prolog programs that is tightly integrated within an overall assertionbased program development scheme. Our starting point is the Ciao model, a framework that unifies unit testing and run-time verification, as well as static verification and static debugging, using a common assertion language. Properties which cannot be verified statically are checked dynamically. In this context, the idea of generating random test values from assertion preconditions emerges naturally since these preconditions are conjunctions of literals, and the corresponding predicates can in principle be used as generators. Our tool generates valid inputs from the properties that appear in the assertions shared with other parts of the model, and the run time-check instrumentation of the Ciao framework is used to perform a wide variety of checks. This integration also facilitates the combination with static analysis. The generation process is based on running standard predicates under non-standard (random) search rules. Generation can be fully automatic but can also be guided or defined specifically by the user. We propose methods for supporting (C)LP-specific properties, including combinations of shape-based (regular) types and variable sharing and instantiation, and we also provide some ideas for shrinking for these properties. We also provide a case study applying the tool to the verification and checking of the code of some of the abstract domains used by the Ciao system.
\end{abstract}

\section{Introduction and Motivation}

Code validation is a vital task in any software development cycle. Traditionally, two of the main approaches used to this end are verification and testing. The former uses formal methods to prove automatically or interactively some specification of the code, while the latter mainly consists in executing the code for concrete inputs or test cases and checking that the program input-output relations (and behaviour, in general) are the expected ones.

Research partially funded by MINECO TIN2015-67522-C3-1-R TRACES project, and the Madrid P2018/TCS-4339 BLOQUES-CM program. We are also grateful to the anonymous reviewers for their useful comments. 
The Ciao language [11] introduced a novel development workflow [12, 13,21] that integrates the two approaches above. In this model, program assertions are fully integrated in the language, and serve both as specifications for static analysis and as run-time check generators, unifying run-time verification and unit testing with static verification and static debugging. This model represents an alternative approach for writing safe programs without relying on full static typing, which is specially useful for dynamic languages like Prolog, and can be considered an antecedent of the popular gradual- and hybrid-typing approaches $[5,22,24]$.

The Ciao Model: For our purposes, assertions in the Ciao model can be seen as a shorthand for defining instrumentation to be added to programs, in order to check dynamically preconditions and postconditions, including conditional postconditions, properties at arbitrary program points, and certain computational (non-functional) properties. The run-time semantics implemented by the translation of the assertion language ensures that execution paths that violate the assertions are captured during execution, thus detecting errors. Optionally, (abstract interpretation-based [4]) compile-time analysis is used to detect assertion violations, or to prove (parts of) assertions true, verifying the program or reducing run-time checking overhead. As an example, consider the following $\mathrm{Ci}$ ao code (with the standard definition of quick-sort):

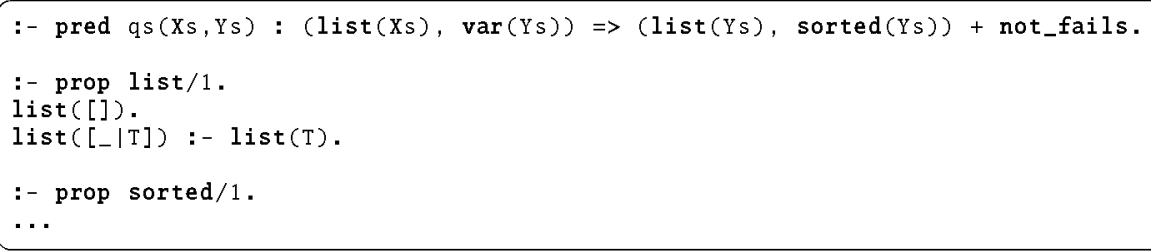

The assertion has a calls field (the conjunction after ':'), a success field (the conjunction after '=>'), and a computational properties field (after ' + '), where all these fields are optional. It states that a valid calling mode for $q s / 2$ is to invoke it with its first argument instantiated to a list, and that it will then return a list in Ys, that this list will be sorted, and that the predicate will not fail. Properties such as list/1 or sorted/1 are normal predicates, but which meet certain conditions (e.g., termination) [13] and are marked as such via prop/1 declarations. Other properties like var/1 or not_fails are builtins.

Compile-time analysis with a types/shapes domain can easily detect that, if the predicate is called as stated in the assertion, the list (Ys) check on success will always succeed, and that the predicate itself will also succeed. If this predicate appears within a larger program, analysis can also typically infer whether or not qs/2 is called with a list and a free variable. However, perhaps, e.g., sorted(Ys) cannot be checked statically (this is in fact often possible, but let us assume that, e.g., a suitable abstract domain is not at hand). The assertion would then be simplified to:

:- pred qs (Xs, Ys) $\Rightarrow$ sorted (Ys). 
At run time, sorted(Ys) will be called within the assertion checking-harness, right after calls to qs $/ 2$. This harness ensures that the variable bindings (or constraints) and the whole checking process are kept isolated from the normal execution of the program (this can be seen conceptually as including a Prolog copy_term, or calling within a double negation, $\backslash+\backslash+$, executing in a separate process, etc.).

Testing vs. Run-Time Checking: The checking of sorted/1 in the example above will occur in principle during execution of the program, i.e., in deployment. However, in many cases it is not desirable to wait until then to detect errors. This is the case for example if errors can be catastrophic or perhaps if there is interest in testing, perhaps for debugging purposes, more general properties that have not been formally proved and whose main statements are not directly part of the program (and thus, will never be executed), such as, e.g.:

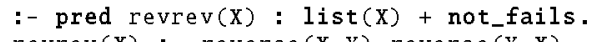

This implies performing a testing process prior to deployment. The Ciao model includes a mechanism, integrated with the assertion language, that allows defining test assertions, which will run (parts of) the program for a given input and check the corresponding output, as well as driving the run-time checks independently of concrete application data [16]. For example, if the following (unit) tests are added to qs/2:

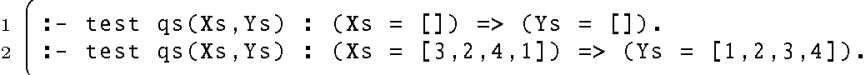

qs $/ 2$ will be run with, e.g., $[3,2,4,1]$ as input in Xs, and the output generated in Ys will be checked to be instantiated to $[1,2,3,4]$. This is done by extracting the test drivers [16]:

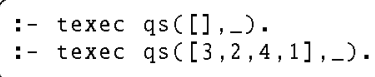

and the rest of the work (checking the assertion fields) is done by the standard assertion run-time checking machinery. In our case, this includes checking at run time the simplified assertion ":- pred qs(Xs,Ys) $\Rightarrow>\operatorname{sorted(Ys).",~so~that~}$ the output in Ys will be checked by calling the implementation of sorted/1. This overall process is depicted in Fig. 1 and will be discussed further in Sect. 2.

Towards Automatic Generation: Hand-written test cases such as those above are quite useful in practice, but they are also tedious to write and even when they are present they may not cover some interesting cases. An aspect that is specific to (Constraint-)Logic Programming (CLP) and is quite relevant in this context is that predicates in general (and properties in particular) can be used as both checkers and generators. For example, calling list $(\mathrm{X})$ from the 
revrev/1 example above with $\mathrm{X}$ uninstantiated generates lazily, through backtracking, an infinite set of lists, $\mathrm{Xs}=[] ; \mathrm{Xs}=\left[_{-}\right] ; \mathrm{Xs}=\left[_{-},{ }_{-},{ }_{-}\right] \ldots$, which can be used to catch cases in which an error in the coding of reverse $/ 2$ makes revrev/ 1 fail. This leads naturally to the idea of generating systematically and automatically test cases by running in generation mode (i.e., "backwards") the properties in the calls fields of assertions.

While this idea of using properties as test case generators has always been present in the descriptions of the Ciao model [12,21], it has not really been exploited significantly to date. Our purpose in this paper is to fill this gap. We report on the development of LPtest, an implementation of random testing [8] with a more natural connection with Prolog semantics, as well as with the Ciao framework. Due to this connection and the use of assertions, this assertion-based testing allows supporting complex properties like combinations of shape-based (regular) types, variable sharing, and instantiation, and also non-functional properties.

Our contributions can be summarized as follows:

- We have developed an approach and a tool for assertion-based random test generation for Prolog and related languages. It has a number of characteristics in common with property-based testing from functional languages, as exemplified by QuickCheck [3], but provides the assertions and properties required in order to cover (C)LP features such as logical variables and nonground data structures or non-determinism, with related properties such as modes, variables sharing, non-failure, determinacy and number of solutions, etc. In this, LPtest is most similar to PrologTest [1], but we argue that our framework is more general and we support richer properties.

- Our approach offers a number of advantages that stem directly from framing it within the $\mathrm{Ciao}$ model. This includes the integration with compile-time checking (static analysis) and the combination with the run-time checking framework, etc. using a single assertion language. This for example greatly simplifies error reporting and diagnosis, which can all be inherited from these parts of the framework. It can also be combined with other test-case generation schemes. To the extent of our knowledge, this has only been attempted partially by PropEr [20]. Also, since Erlang is in many ways closer to a functional language, PropEr does not support Prolog-relevant properties and it is not integrated with static analysis. In comparison to PrologTest, we provide combination with static analysis, through an integrated assertion language, whereas the assertions of PrologTest are specific to the tool, and we also support a larger set of properties.

- In our approach the automatic generation of inputs is performed by running in generation mode the properties (predicates) in those preconditions, taking advantage of the specialized SLD search rules of the language (e.g., breadth first, iterative deepening, and, in particular, random search) or implementations specialized for such generation. In particular, we perform automatic generation of instances of Prolog regular types, instantiation modes, sharing relations among variables and grounding, arithmetic constraints, etc. 


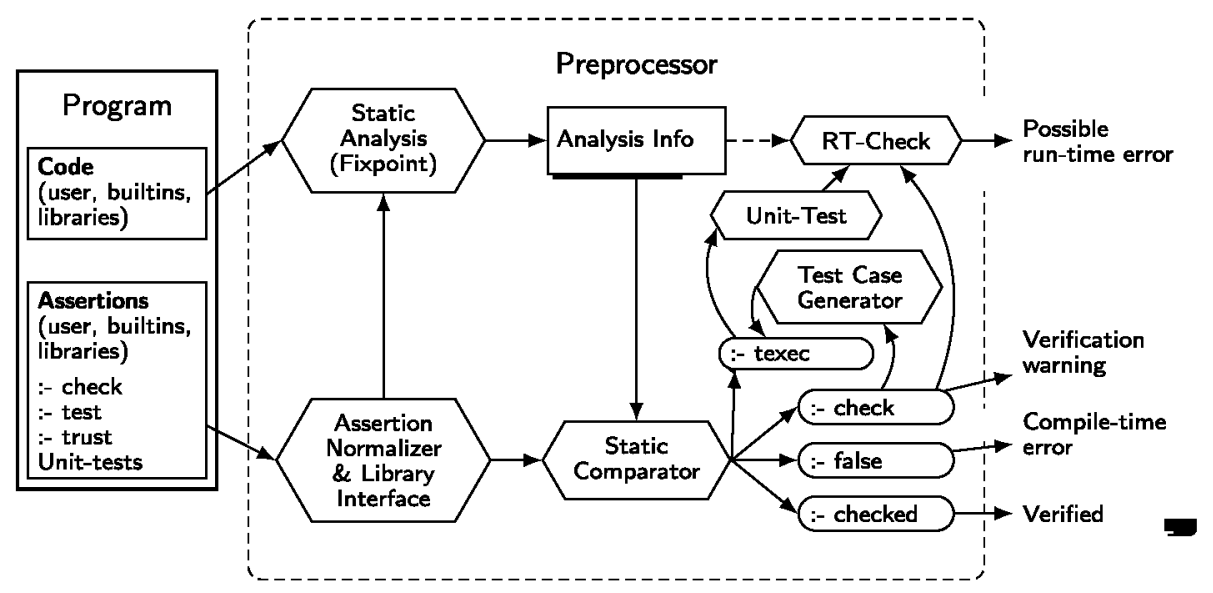

Fig. 1. The Ciao assertion framework (CiaoPP's verification/testing architecture). (Color figure online)

To the extent of our knowledge all previous tools only supported generation for types, while we also consider the latter.

- We have enhanced assertion and property-based test generation by combining it with static analysis and abstract domains. To the extent of our knowledge previous work had at most discarded properties that could be proved statically (which in LPtest comes free from the overall setting, as mentioned before), but not used static analysis information to guide or improve the testing process.

- We have implemented automatic shrinking for our tool, and in particular we have developed an automatic shrinking algorithm for Prolog regular types.

The rest of this paper is organized as follows. In Sect. 2 we review our approach to assertion-based testing in the context of Prolog and Ciao. In Sect. 3 we introduce our test input generation schema. In Sect. 4, we show how assertionbased testing can be combined with and enhanced by static analysis. Sect. 5 is dedicated to shrinking of test cases in LPtest. In Sect. 6 we show some preliminary results of a case study in which our tool is applied to checking some of the domain operations in the static analyses of CiaoPP (the Ciao "preprocessor"). Finally, we review the related work in Sect. 7 and provide our conclusions in Sect. 8 .

\section{Using LPtest Within the Ciao Model}

As mentioned before, the goal of LPtest is to integrate random testing of assertions within Ciao's assertion-based verification and debugging framework (Fig. 1). Given an assertion for a predicate, we want to generate goals for that predicate satisfying the assertion precondition (i.e., valid call patterns for the 
predicate) and execute them to check that the assertion holds for those cases or find errors. As also mentioned in the introduction, the Ciao framework already provides most of the components needed for this task: the run-time checking framework allows us to check at runtime that the assertions for a predicate are not violated, and the unit-test framework allows us to specify and run concrete goals to check those assertions. We only need to be able to generate terms satisfying the assertion preconditions and feed them into the other parts of the framework (the new yellow box in Fig. 1). This generation of test cases is discussed in Sect. 3, and the following example shows how everything is integrated step by step.

Consider again a similar assertion for the qs/2 predicate, and assume that the program has a bug and fails for lists with repeated elements:

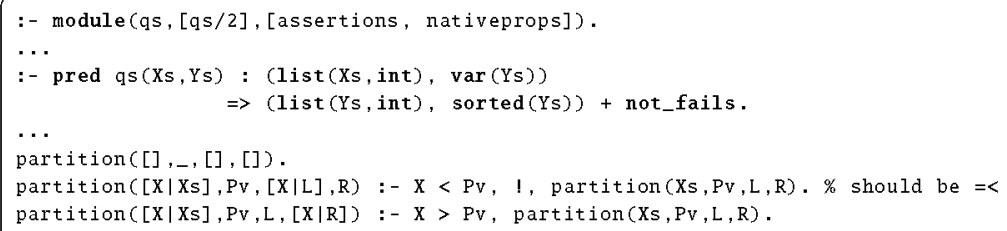

Following Fig. 1, the assertions of the qs module are verified statically [13]. As a result, parts of each assertion may be proved true or false (in which case no testing is needed for them), and, if any other parts are left after this process, run-time checking and/or testing is performed for them. Ci aoPP generates a new source file which includes the original assertions marked with status checked, false, or, for the ones that remain for run-time checking, check. LPtest starts by reporting a simple adaptation of CiaoPP's output. E.g., for our example, LPtest will output:

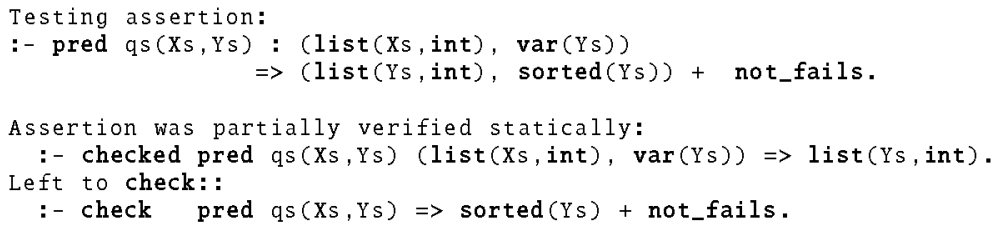

LPtest will then try to test dynamically the remaining assertion. For that, it will first collect the Ciao properties that the test case must fulfill (i.e., those in the precondition of the assertion, which is taken from the original assertion, which is also output by (iaoPP), and generate a number of test case drivers (texec's) satisfying those properties. Those test cases will be pipelined to the unit-test framework, which, relying on the standard run-time checking instrumentation, will manage their execution, capture any error reported during runtime checking, and return them to LPtest, which will output: 


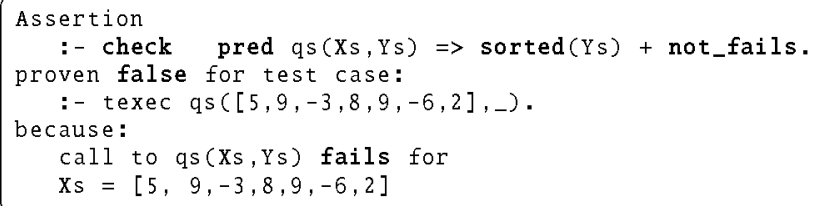

Finally, LPtest will try to shrink the test cases, enumerating test cases that are progressively smaller and repeating the steps above in a loop to find the smallest test case which violates the assertion. LPtest will output:

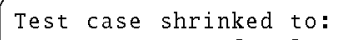

The testing algorithm for a module can thus be summarized as follows:

1. (CiaoPP) Use static analysis to check the assertions. Remove proved assertions, simplify partially proved assertions.

2. (LPtest) For each assertion, generate $N$ test cases from the properties in the precondition, following the guidelines in Sect. 3. For each test case, go to 3 . Then go to 4 .

3. (RTcheck) Use the unit-test framework to execute the test case and capture any run-time checking error (i.e., assertion violation).

4. (LPtest) Collect all failed test cases from RTcheck. For each of them, go to $\mathbf{5}$ to shrink them, and then report them (using RTcheck).

5. (LPtest) Generate a simpler test case not generated yet.

- If not possible, finalize and return current test case as shrinked test case. If possible, go to 3 to run the test.

* If the new test case fails, go to $\mathbf{5}$ with the new test case.

* If it succeeds, repeat this step.

The use of the Ciao static verification and run-time checking framework in this (pseudo-)algorithm, together with the rich set of native properties in Ciao, allows us to specify and check a wide range of properties for our programs. We provide a few examples of the expressive power of the approach:

(Conditional) Postconditions. We can write postconditions using the success $\Leftrightarrow>$ field of the assertions. Those postconditions can range from user-defined predicates to properties native to CiaoPP, for which there are built-in checkers in the run-time checking framework. These properties include types, which can be partially instantiated, i.e., contain variables, and additional features particular to logic programming such as modes and sharing between variables. As an example, one can test with LPtest the following assertions, where covered( $\mathrm{X}, \mathrm{Y})$ means that all variables occurring in $\mathrm{X}$ also occur in $\mathrm{Y}$ : 


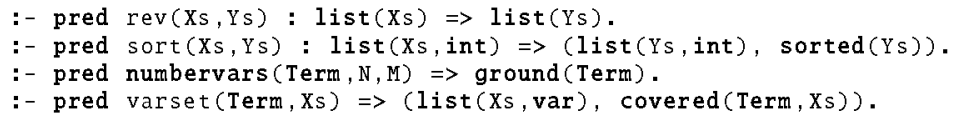

For this kind of properties, LPtest tries to ensure that at least some of the test cases do not succeed trivially (by the predicate just failing), and warns otherwise.

Computational Properties. LPtest can also be used to check properties regarding the computation of a predicate. These properties are typically native and talk about features that range from determinism and multiplicity of solutions to resource usage (cost). They can be checked with LPtest, as long as the run-time checking framework supports it (e.g., some properties, like termination, are not decidable). Examples of this would be:

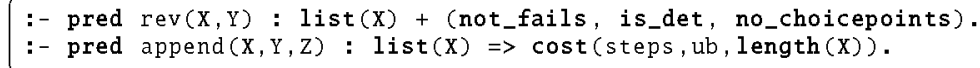

Rich Generation. The properties supported for generation include not only types, but also modes and sharing between variables, and arithmetic constraints, as well as a restricted set of user-defined properties. As an example, LPtest can test the following assertion:

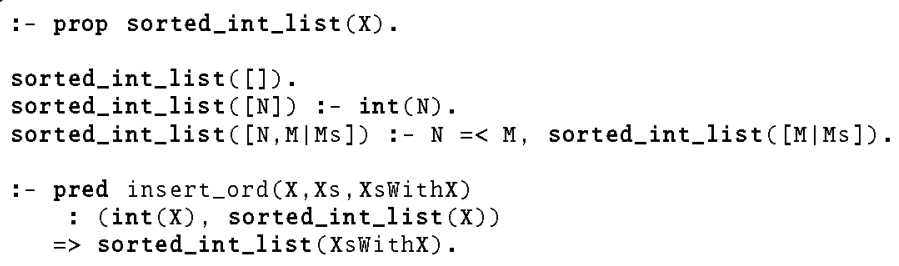

\section{Test Case Generation}

The previous section illustrated specially the parts that LPtest inherits from the Ciao framework, but a crucial step was skipped: the generation of test cases from the calls field of the assertions, i.e., the generation of Prolog terms satisfying a conjunction of $\mathrm{Ci}$ ao properties. This was obviously one of the main challenges we faced when designing and implementing LPtest. In order for the tool to be integrated naturally within the Ciao verification and debugging framework, this generation had to be as automatic as possible. However, full automation is not always possible in the presence of arbitrary properties potentially using the whole Prolog language (e.g., cuts, dynamic predicates, etc.). The solution we arrived at is to support fully automatic and efficient generation for reasonable subsets of the Prolog language, and provide means for the user to guide the generation in more complex scenarios. 
Pure Prolog. The simplest and essential subset of Prolog is pure Prolog. In pure Prolog every predicate, and, in particular, every Ciao property, is itself a generator: if it succeeds with some terms as arguments, those terms will be (possibly instances of) answers to the predicate when called with free variables as arguments. The problem is that the classic depth-first search strategy used in Prolog resolution, with which those answers will be computed, is not well suited for test-case generation. One of Ciao's features comes here to the rescue. Ciao has a concept of packages, syntactic and/or semantic extensions to the language that can be loaded module-locally. This mechanism is used to implement language extensions such as functional syntax, constraints, higher order, etc., and, in particular alternative search rules. These include for example (several versions of) breadth first, iterative deepening, Andorra-style execution, etc. These rules can be activated on a per-module basis. For example, the predicates in a module that starts with the following header:

:- module( myprops, _, $[b f]$ ).

(which loads the bf package) will run in breadth-first mode. While breadthfirst is useful mostly for teaching, other alternative search rules are quite useful in practice. Motivated by the LPtest context, i.e., with the idea of running properties in generation mode, we have developed also a randomized alternative search strategy package, rnd, which can be described by the following simplified meta-interpreter:

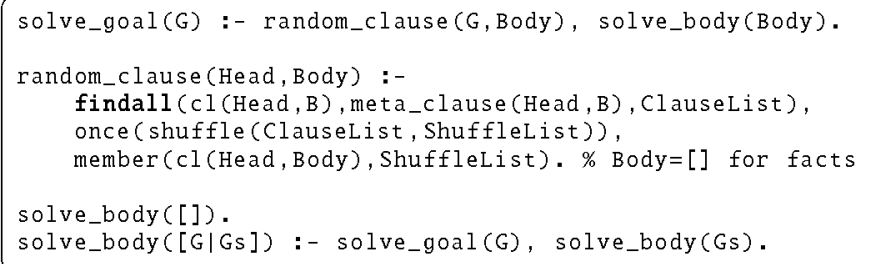

The actual algorithm used for generation is of course more involved. Among other details, it only does backtracking on failure (on success it starts all over again to produce the next answer, without repeating traces), and it has a growth control mechanism to avoid getting stuck in traces that lead to non-terminating generations.

Using this search strategy, a set of terms satisfying a conjunction of pure Pro$\log$ properties can be generated just by running all those properties sequentially with unbounded variables. This is implemented using different versions of each property (generation, run-time check) which are generated automatically from the declarative definition of the property using instrumentation. In particular, this simplest subset of the language allows us to deal directly with regular types (e.g., list/1).

Mode, Sharing, and Arithmetic Constraints. We extend the subset of the language for which generation is supported with arithmetic (e.g., int/1, flt/1, 
$</ 2$ ), mode-related extralogical predicates and properties (e.g., free/1, gnd/1), and sharing-related native properties (e.g., mshare/1, which describes the sharing - aliasing- relations of a set of variables using sharing sets [15], and indep/2, that states that two variable do not share). When a goal or a property of this kind appears during generation, the variables occurring in it are constrained using a constraints domain. The domain ensures that those constraints are satisfiable during all steps of generation, failing and backtracking otherwise. There is a last step in generation in which all free variables are randomly further intantiated in a way that those constraints are satisfied.

This can be seen conceptually as choosing first a trace at random for each property and collecting constraints in the trace, and then randomly sampling (enumerating) the constrains. However, since the constraints introduced by unification are terms, it is equivalent to solving a predicate with the random search strategy and treating each builtin or native property as a constraint. In practice, we support more builtins for generation in properties (e.g., ==/2 just unifies two variables, we have shape constraints that handle $=. / 2$, and support negation to some extent), but the approach has only been tested significantly for the subset of Prolog presented so far.

In the last phase of constraints (random sampling), unconstrained free variables can be further instantiated with some probability, using random shape and sharing constraints, chosen among native properties and properties defined by the users on modules that are loaded at the time. This way, random terms are still generated for an assertion without precondition, or the generated term for list $(X)$ is not always a list of free variables. This is also the technique used to further instantiate a free variable constrained as ground but for which no shape information is available.

Generation for Other Properties. For the remaining properties which use Prolog features not covered so far (e.g., dynamic predicates), there is a last step in the generation algorithm in which they are simply checked for the terms generated so far. User-defined generators are encouraged for assertions with preconditions that are complex enough to reach this step. There is a limit to how many times generation can reach this step and fail, to avoid getting stuck in an inefficient or non-terminating generate-and-check loop. To recognize these properties without inspecting the code (left as future work), users are trusted to mark the properties suitable for generation, and only the native properties discussed and the regular types are considered suitable by default.

\section{Integration with Static Analysis}

The use of a unified assertion framework for testing and analysis allows us to enhance LPtest random testing by combining it with static analysis.

First of all, as illustrated in Sect. 2 and Fig. 1, Ci aoPP first performs a series of static analyses through which some of the assertions may be verified statically, possibly partially. Thus, only some parts of some assertions may need to be checked in the testing phase [13]. 
Beyond this, and perhaps more interestingly in our context, statically inferred information can also help while testing the remaining assertions. In particular, it is used to generate more relevant test cases in the generation phase. Consider for example the following assertion:

:- pred qs(Xs,Ys) $\Rightarrow$ sorted (Ys).

Without the usual precondition, LPtest would have to generate arbitrary terms to test the assertion, most of which would not be relevant test cases since the predicate would fail for them, and therefore the assertion would be satisfied trivially. However, static analysis typically infers the output type for this predicate:

:- $\operatorname{pred} q s(X s,)^{\prime} \Rightarrow$ list $(X s$, int $)$.

I.e., analysis infers that on success Xs must be a list, and so on call it must be compatible with a list if it is to succeed (inputs that generate failure are also interesting of course, but not to check properties that should hold on success). Therefore the assertion can also be checked as follows:

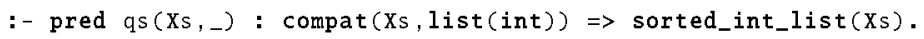

where compat(Xs, list(int)) means that Xs is either a list of integers or can be further instantiated to one. Now we would only generate relevant inputs (generation for compat/ 2 is implemented by randomly uninstantiating a term), and LPtest is able to prove the assertion false. The same can be done for modes and sharing to some extent: variables that are inferred to be free on success must also be free on call, and variables inferred to be independent must be independent on call too. Also, when a predicate is not exported, the calls assertions inferred for it can be used for generation. In general, the idea here is to perform some backwards analysis. However, this can also be done without explicit backwards analysis by treating testing and (forward) static analysis independently and one after the other, which makes the integration conceptually simple and easy to implement.

A Finer-Grain Integration. We now propose a finer-grained integration of assertion-based testing and analysis, which still treats analysis as a black box, although not as an independent step. So far our approach has been to try to check an assertion with static analysis, and if this fails we perform random testing. However, the analysis often fails to prove the assertion because its precondition (i.e., the entry abstract substitution to the analysis) is too general, but it can prove it for refinements of that entry, i.e., refinements of the precondition. In that case, all test cases satisfying that refined precondition are guaranteed to succeed, and therefore useless in practice. We propose to work with different refined versions of an assertion, by adding further, exhaustive constraints in a 
native domain to the precondition, and performing testing only on the versions which the analysis cannot verify statically, thus pruning the test case input space. For example, for an assertion of a predicate of arity one, without mode properties, a set of assertions equivalent to the original one would result by generating three different assertions with the same success but preconditions ground $(X), \operatorname{var}(X)$, and (nonground $(X)$, nonvar $(X)$ ). The idea is to generalize this to arbitrary, maybe infinite abstract domains, for which a given abstract value is not so easily partitioned as in the example above. Alternatively, the test exploration can be limited to subsets of the domain, since in any case the testing process cannot achieve completeness in general. The core of an algorithm for this domain partition would be the following: to test an assertion for a given entry $A \in D_{\alpha}$, the assertion is proved by the analysis or tested recursively for a set of abstract values $S \subseteq\left\{B \mid B \in D_{\alpha}, B \sqsubseteq A\right\}$ lower than that entry, and random test cases are generated in the "space" between the entry and those lower values $\gamma(A) \backslash \bigcup \gamma(B)$, where $\gamma$ is the concretization function in the domain. For this it is only necessary to provide a suitable sampling function in the domain, and a rich generation algorithm for that domain. But note that, e.g., for the sharingfreeness domain, we already have the latter: we already have generation for mode and sharing constraints, and a transformation scheme between abstract values and mode/sharing properties. Note also that all this can still be done while treating the static analysis as a black box, and that if the enumeration of abstract values is fine-grained enough, this algorithm also ensures coverage of the test input space during generation.

\section{$5 \quad$ Shrinking}

One flaw of random testing is that often the failed test cases reported are unnecessary complex, and thus not very useful for debugging. Many property-based tools introduce shrinking to solve this problem: after one counter-example is found, they try to reduce it to a simpler counter-example that still fails the test in the same way. LPtest supports shrinking too, both user-guided and automatic. We present the latter.

The shrinking algorithm mirrors that of generation, and in fact reuses most of the generation framework. It can be seen as a new generation with further constraints: bounds on the shape and size of the generated goal. The traces followed to generate the new term from a property must be "subtraces" of the ones followed to generate the original one. The random sampling of the constraints for the new terms must be "simpler" than for the original ones. The final step in which the remaining properties are checked is kept.

We present the algorithm for the first step. Generation for the shrinked value follows the path that leads to the to-be-shrinked value, but at any moment it can non-deterministically stop following that trace and generate a new subterm using size parameter 0. Applying this method to shrink lists of Peano numbers is equivalent to the following predicate, where the first argument is the term to be shrinked and the second a free variable to be the shrinked value on success: 


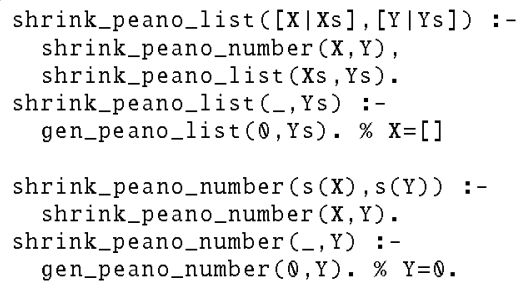

This method can shrink the list $[s(\theta), \theta, s(s(s(\theta)))]$ to $[s(\theta), \theta]$ or $[s(\theta), \theta, s(s(\theta))]$, but never to $[s(\theta), s(s(s(\theta)))]$. To solve that, we allow the trace of the to-be-shrinked term to advance non-deterministically at any moment to an equivalent point, so that the trace of the generated term does not have to follow it completely in parallel. It would be as if the following clauses were added to the the previous predicate (the one which sketches the actual workings of the method during meta-interpretation):

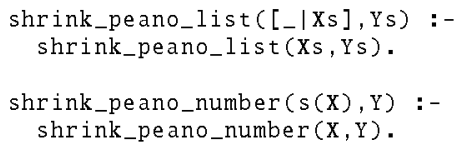

With this method, $[s(\theta), s(s(s(\theta)))]$ would now be a valid shrinked value.

This is implemented building shrinking versions of the properties, similarly to the examples presented, and running them in generation mode. However, since we want shrinking to be an enumeration of simpler values, and not random, the search strategy used is the usual depth-first strategy and not the randomized one presented in Sect. 3. The usual sampling of constraints is used too, instead of the random one.

The number of potential shrinked values grows exponentially with the size of the traces. To mitigate this problem, LPtest commits to a shrinked value once it checks that it violates the assertion too, and continues to shrink that value, but never starts from another one on backtracking. Also, the enumeration of shrinked values returns first the values closer to the original term, i.e., if $\mathbf{X}$ is returned before $\mathrm{Y}$, then shrinking $\mathrm{Y}$ could never produce $\mathrm{X}$. Therefore we never repeat a shrinked value ${ }^{1}$ in our greedy search for the simplest counterexample.

\section{A Case Study}

In order to better illustrate our ideas, we present now a case study which consists in testing the correctness of the implementation of some of CiaoPP's abstract domains. In particular, we focus herein on the sharing-freeness domain [19] and

1 Actually, we do not repeat subtraces, but two different subtraces can represent the same value (e.g., there are two ways to obtain $\mathrm{s}(0)$ from $\mathrm{s}(\mathrm{s}(0))$ ). 
the correctness of its structure as a lattice and its handling of builtins. Tested predicates include leq/ 2 , which checks if an abstract value is below another in the lattice, lub/3 and glb/3, which compute the least upper bound and greatest lower bound of two abstract values, builtin_success/3, which computes the success substitution of a builtin from a call substitution, and abstract $/ 2$, which computes the abstraction for a list of substitutions.

Generation. Testing these predicates required generating random values for abstract values and builtins. The latter is simple: a simple declaration of the property builtin(F,A), which simply enumerates the builtins together with their arity, is itself a generator, and using the generation scheme proposed in Sect. 3 it becomes a random generator, while it can still be used as a checker in the run-time checking framework. The same happens for a simple declarative definition of the property $\operatorname{shfr}(\mathrm{ShFr}, \mathrm{Vs}$ ), which checks that $\mathrm{ShFr}$ is a valid sharing-freeness value for a list of variables Vs. This is however not that trivial and proves that our generation scheme works and is useful in practice, since that property is not a regular type, and among others it includes sharing constraints between free variables. These two properties allowed us to test assertions like the following:

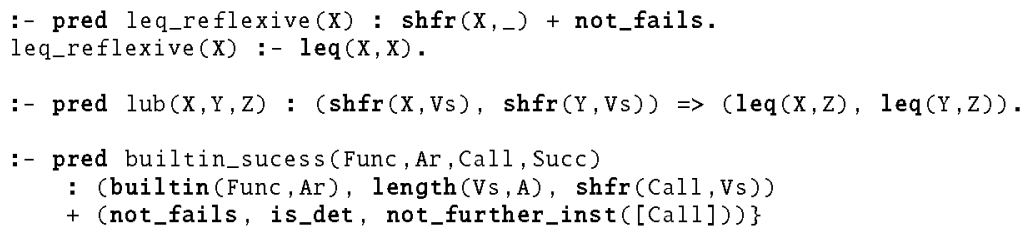

To check some assertions we needed to generate related pairs of abstract values. That is encoded in the precondition as a final literal leq ( $\operatorname{ShFr} 1, \operatorname{ShFr} 2)$, as in the next assertion:

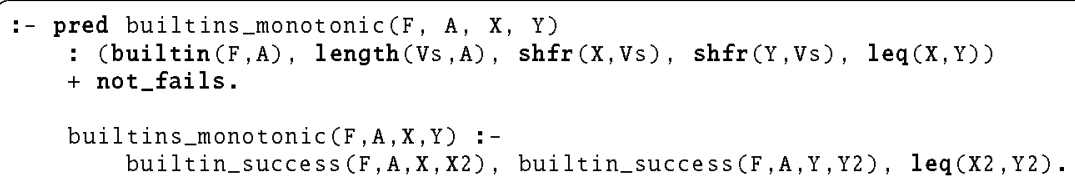

In our framework the generation is performed by producing first the two values independently, and checking the literal. This became inefficient, so we decided to write our own generator for this particular case. Finally, we tested the generation for arbitrary terms with the following assertion, which checks that the abstract value resulting from executing a builtin and abstracting the arguments on success is lower than the one resulting of abstracting the arguments on call and calling builtin_success/3: 


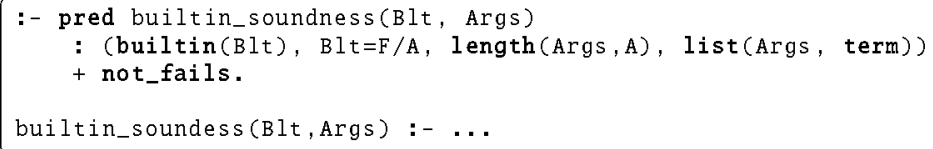

Analysis. Many properties used in our assertions were user-defined, complex, and not native to $\mathrm{Ci}$ aoPP, so there were many cases in which the analysis could not abstract them precisely. However, the analysis did manage to simplify or prove some of the remaining ones, particularly regular types and those dealing with determinism (+ is_det) and efficiency (no_choicepoints). Additionally, we successfully did the experiment of not defining the regular type builtin/2, and letting the analysis infer it on its own and use it for generation. We also tested by hand the finer integration between testing and analysis proposed in Sect. 4: some assertions involving builtins could not be proven for the general case, but this could be done for some of the simpler builtins, and thus testing could be avoided for those particular cases.

Bugs Found. We did not find any bugs in the implementations for different domains of the lattice operations 1 eq $/ 2,1 \mathrm{ub} / 2$, and $\mathrm{glb} / 2$. This was not surprising: they are relatively simple and commonly used in CiaoPP. However, we found several bugs in builtin_success/2 (part of the description of the "transfer function" for some language built-ins) in some domains. Some of them were minor and thus had never been found or reported before: some builtin handlers left unnecessary choicepoints, or failed for the abstract value $\perp$ (with which they are never called in CiaoPP). Others were more serious: we found bugs for less commonly-used builtins, and even two larger bugs for the builtins $=/ 2$ and $==/ 2$. The handler failed for the literal $X=X$ and for literals like $f(X)==g(Y)$, both of which do not normally appear in realistic programs and thus were not detected before.

\section{Related Work}

Random testing has been used for a long time in Software Engineering [8]. As mentioned before, the idea of using properties and assertions as test case generators was proposed in the context of the Ciao model [12,21] for logic programs, although it had not really been exploited significantly until this work. QuickCheck [3] provided the first full implementation of a property-based random test generation system. It was first developed for Haskell and functional programming languages in general and then extended to other languages, and has seen significant practical use [14]. It uses a domain-specific language of testable specifications and generates test data based on Haskell types. ErlangQuickCheck and PropEr [20] are closely related systems for Erlang, where types are dynamically checked and the value generation is guided by means of functions, using quantified types defined by these generating functions. We use a number of ideas 
from QuickCheck and the related systems, such as applying shrinking to reduce the test cases. However, LPtest is based on the ideas of the (earlier) Ciao model and we do not propose a new assertion language, but rather use and extend that of the Ci ao system. This allows supporting Prolog-relevant properties, which deal with non-ground data, logical variables, variable sharing, etc., while Qui ckCheck is limited to ground data. Also, while QuickCheck offers quite flexible control of the random generation, we argue that using random search strategies over predicates defining properties is an interesting and more natural approach for Prolog.

The closest related work is PrologTest [1], which adapts QuickCheck and random property-based testing to the Prolog context. We share many objectives with PrologTest but we argue that our framework is more general, with richer properties (e.g., variable sharing), and is combined with static analysis. Also, as in QuickCheck, PrologTest uses a specific assertion language, while, as mentioned before, we share the $\mathrm{Cia}$ assertions with the other parts of the Ciao system. PrologTest also uses Prolog predicates as random generators. This can also be done in LPtest, but we also propose an approach which we argue is more elegant, based on separating the code of the generator from the random generation strategy, using the facilities present in the Ciao system for running code under different SLD search rules, such as breadth first, iterative deepening, or randomized search.

Other directly related systems are EasyCheck [2] and CurryCheck [9] for the Curry language. In these systems test cases are generated from the (strong) types present in the language, as in QuickCheck. However, they also deal with determinism and modes. To the extent of our knowledge test case minimization has not been implemented in these systems.

There has also been work on generating test cases using CLP and partial evaluation techniques, both for Prolog and imperative languages (see, e.g., $[6,7]$ and its references). This work differs from (and is complementary to) ours in that the test cases are generated via a symbolic execution of the program, with the traditional aims of path coverage, etc., rather than from assertions and with the objective of randomized testing.

Other related work includes fuzz testing [18], where "nonsensical" (i.e., fully random) inputs are passed to programs to trigger program crashes, and grammar-based testing, where inputs generation is based on a grammatical definition of inputs (similar to generating with regular types) [10]. Schrijvers proposed Tor [23] as a mechanism for supporting the execution of predicates using alternative search rules, similar in spirit to Ciao's implementation of searchstrategies via packages. Midtgaard and Moller [17] have also applied propertybased testing to checking the correctness of static analysis implementations.

\section{Conclusions and Future Work}

We have presented an approach and a tool, LPtest, for assertion-based random testing of Prolog programs that is integrated with the Ciao assertion model. 
In this context, the idea of generating random test values from assertion preconditions emerges naturally since preconditions are conjunctions of literals, and the corresponding predicates can conceptually be used as generators. LPtest generates valid inputs from the properties that appear in the assertions shared with other parts of the model. We have shown how this generation process can be based on running the property predicates under non-standard (random) search rules and how the run time-check instrumentation of the Ciao framework can be used to perform a wide variety of checks. We have proposed methods for supporting (C)LP-specific properties, including combinations of shape-based (regular) types and variable sharing and instantiation. We have also proposed some integrations of the test generation system with static analysis and provided a number of ideas for shrinking in our context. Finally, we have shown some results on the applicability of the approach and tool to the verification and checking of the implementations of some of Ciao's abstract domains. The tool has already proven itself quite useful in finding bugs in production-level code.

\section{References}

1. Amaral, C., Florido, M., Santos Costa, V.: PrologCheck - property-based testing in prolog. In: Codish, M., Sumii, E. (eds.) FLOPS 2014. LNCS, vol. 8475, pp. 1-17. Springer, Cham (2014). https://doi.org/10.1007/978-3-319-07151-0_1

2. Christiansen, J., Fischer, S.: EasyCheck - test data for free. In: Garrigue, J., Hermenegildo, M.V. (eds.) FLOPS 2008. LNCS, vol. 4989, pp. 322-336. Springer, Heidelberg (2008). https://doi.org/10.1007/978-3-540-78969-7_23

3. Claessen, K., Hughes, J.: QuickCheck: a lightweight tool for random testing of Haskell programs. In: Fifth ACM SIGPLAN International Conference on Functional Programming, ICFP 2000, pp. 268-279. ACM (2000)

4. Cousot, P., Cousot, R.: Abstract interpretation: a unified lattice model for static analysis of programs by construction or approximation of fixpoints. In: Proceedings of POPL 1977, pp. 238-252. ACM Press (1977)

5. Flanagan, C.: Hybrid type checking. In: 33rd ACM SIGPLAN-SIGACT Symposium on Principles of Programming Languages, POPL 2006, pp. 245-256, January 2006

6. Gómez-Zamalloa, M., Albert, E., Puebla, G.: On the generation of test data for prolog by partial evaluation. In: Proceedings of WLPE 2008, pp. 26-43 (2008)

7. Gómez-Zamalloa, M., Albert, E., Puebla, G.: Test case generation for objectoriented imperative languages in CLP. Theor. Pract. Logic Prog. 10(4-6), 659-674 (2010). ICLP 2010 Special Issue

8. Hamlet, D.: Random testing. In: Marciniak, J. (ed.) Encyclopedia of Software Engineering, pp. 970-978. Wiley, New York (1994)

9. Hanus, M.: CurryCheck: checking properties of curry programs. In: Hermenegildo, M.V., Lopez-Garcia, P. (eds.) LOPSTR 2016. LNCS, vol. 10184, pp. 222-239. Springer, Cham (2017). https://doi.org/10.1007/978-3-319-63139-4_13

10. Hennessy, M., Power, J.F.: An analysis of rule coverage as a criterion in generating minimal test suites for grammar-based software. In: 20th IEEE/ACM International Conference on Automated Software Engineering (ASE 2005), pp. 104-113, November 2005 
11. Hermenegildo, M.V., et al.: An overview of ciao and its design philosophy. TPLP 12(1-2), 219-252 (2012). http://arxiv.org/abs/1102.5497

12. Hermenegildo, M.V., Puebla, G., Bueno, F.: Using global analysis, partial specifications, and an extensible assertion language for program validation and debugging. In: Apt, K.R., Marek, V.W., Truszczynski, M., Warren, D.S. (eds.) The Logic Programming Paradigm: A 25-Year Perspective, pp. 161-192. Springer, Heidelberg (1999). https://doi.org/10.1007/978-3-642-60085-2_7

13. Hermenegildo, M.V., Puebla, G., Bueno, F., Lopez-Garcia, P.: Integrated program debugging, verification, and optimization using abstract interpretation (and the Ciao system preprocessor). Sci. Comput. Program. 58(1-2), 115-140 (2005). https://doi.org/10.1016/j.scico.2005.02.006

14. Hughes, J.: QuickCheck testing for fun and profit. In: Hanus, M. (ed.) PADL 2007. LNCS, vol. 4354, pp. 1-32. Springer, Heidelberg (2006). https://doi.org/10.1007/ 978-3-540-69611-7_1

15. Jacobs, D., Langen, A.: Accurate and efficient approximation of variable aliasing in logic programs. In: North American Conference on Logic Programming (1989)

16. Mera, E., Lopez-García, P., Hermenegildo, M.: Integrating software testing and run-time checking in an assertion verification framework. In: Hill, P.M., Warren, D.S. (eds.) ICLP 2009. LNCS, vol. 5649, pp. 281-295. Springer, Heidelberg (2009). https://doi.org/10.1007/978-3-642-02846-5_25

17. Midtgaard, J., Møller, A.: QuickChecking static analysis properties. Softw. Test. Verif. Reliab. 27(6) (2017). https://doi.org/10.1002/stvr.1640

18. Miller, B.P., Fredriksen, L., So, B.: An empirical study of the reliability of UNIX utilities. Commun. ACM 33(12), 32-44 (1990). https://doi.org/10.1145/96267. 96279

19. Muthukumar, K., Hermenegildo, M.: Combined determination of sharing and freeness of program variables through abstract interpretation. In: ICLP 1991, pp. 49 63. MIT Press (June 1991)

20. Papadakis, M., Sagonas, K.: A PropEr integration of types and function specifications with property-based testing. In: 10th ACM SIGPLAN Workshop On Erlang, pp. 39-50, September 2011

21. Puebla, G., Bueno, F., Hermenegildo, M.: Combined static and dynamic assertionbased debugging of constraint logic programs. In: Bossi, A. (ed.) LOPSTR 1999. LNCS, vol. 1817, pp. 273-292. Springer, Heidelberg (2000). https://doi.org/10. $1007 / 10720327 \_16$

22. Rastogi, A., Swamy, N., Fournet, C., Bierman, G., Vekris, P.: Safe \& efficient gradual typing for typescript. In: 42nd POPL, pp. 167-180. ACM, January 2015

23. Schrijvers, T., Demoen, B., Triska, M., Desouter, B.: Tor: modular search with hookable disjunction. Sci. Comput. Program. 84, 101-120 (2014)

24. Siek, J.G., Taha, W.: Gradual typing for functional languages. In: Scheme and Functional Programming Workshop, pp. 81-92 (2006) 